\title{
Application of Particle Swarm Optimization in Formulation Design of Pig Feed
}

\author{
Meijuan ZENG a ${ }^{\text {, } 1}$ and Hao LI ${ }^{\text {a }}$ \\ ${ }^{a}$ College of Automation, Wuhan University of Technology, Wuhan, Hubei, China
}

\begin{abstract}
Feed formula design is the core technology of accurate feeding management of livestock and poultry. High quality feed formula can not only improve the comprehensive and effective utilization of feed, give full play to the growth performance of animals, but also reduce production costs, so as to improve the economic benefits of farmers. Due to the characteristics of fast convergence speed and strong optimization ability of particle swarm optimization algorithm, this paper adopts particle swarm optimization algorithm to solve the pig feed formula, and builds the simulation model of the pig feed formula through Matlab to find the optimal solution for the feed formula. At the same time, compared with the traditional linear programming method, the simulation results show that, compared with the traditional linear programming method, the particle swarm optimization algorithm has better optimization results in solving the feed formulation problem.
\end{abstract}

Keywords. feed formulation; particle swarm optimization; linear programming

\section{Introduction}

Feed formula plays a very important role in the livestock and poultry breeding industry. High efficiency and high-quality feed formula can promote the healthy development of the livestock and poultry breeding industry. At present, the traditional manual calculation method has been difficult to meet the needs of actual production, only the computer application technology and feed formula fully combined, in order to better improve the feed conversion rate, shorten the growth cycle of live pigs, so as to reduce economic costs. The traditional computer optimization methods mainly include linear programming, objective programming and fuzzy programming. The lowest cost formula that meets certain constraints is calculated by decision [1]. However, this kind of method lacks flexibility and can only solve some problems with small operation scale, so it is difficult to properly deal with complex feed formulation problems such as multiple constraints or multiple objectives [2].

In recent years, genetic algorithm and ant colony algorithm have also been widely used in the design of feed formula. In reference [3], genetic algorithm was used to carry out feed matching, and the standard genetic algorithm was optimized and improved, so that the algorithm efficiency and formula benefit had achieved better results. Reference [4] adopted the linear programming method, standard genetic algorithm and NSGA-II

${ }^{1}$ Corresponding Author, College of Automation, Wuhan University of Technology, Wuhan, Hubei, China; E-mail: 1250362615@qq.com. 
algorithm to carry out the matching of pig feed, respectively. The comparative analysis showed that the NSGA-II algorithm could not only reduce feed cost, but also effectively reduce ammonia emission, which was more suitable for multi-objective feed formulation problem. Reference [5] applied the continuous space ant colony optimization algorithm to the problem of feed formulation, and demonstrated the effectiveness and practicability of the optimization algorithm through the matching results.

Particle swarm optimization algorithm is widely used in function optimization, image recognition, neural network training and other fields because of its features such as easy implementation, few parameters, fast convergence speed and strong optimization ability [6]. In this paper, we will model and analyze the feed formula for pig feed, take feed production cost as the optimization goal, the nutritional standard required for pig growth period as the constraint, construct the mathematical model of pig feed formula optimization, and solve the feed formula using linear planning method and particle group algorithm respectively, and compare the analysis to show that the particle group algorithm has stronger solving ability.

\section{Pig Feed and Formula Model}

\subsection{Feed Raw Materials and Nutritional Standards}

Feed raw materials in the process of pig growth mainly provides the life-sustaining essential crude protein, amino acids, vitamins, minerals, total calcium, total phosphorus and other nutrients [7], but a single feed is not enough to meet the nutrition needs of pig growth, and according to the nutritional needs of different stages of pig growth will be reasonable matching of different raw materials, not only conducive to the growth and development of pigs, but also improve the utilization rate of feed.

Table 1. Nutritional content and price of feed raw materials (\%).

\begin{tabular}{cccccccccc}
\hline $\begin{array}{c}\text { Nutrient } \\
\text { elements } \\
\text { Ingredients }\end{array}$ & Corn & $\begin{array}{c}\text { Wheat } \\
\text { bran }\end{array}$ & $\begin{array}{c}\text { Soybean } \\
\text { meal }\end{array}$ & $\begin{array}{c}\text { Fish } \\
\text { meal }\end{array}$ & $\begin{array}{c}\text { Cotton } \\
\text { seed } \\
\text { meal }\end{array}$ & $\begin{array}{c}\text { Rapesee } \\
\text { d } \\
\text { dregs }\end{array}$ & $\begin{array}{c}\text { Vegetable } \\
\text { oil }\end{array}$ & $\begin{array}{c}\text { Composite } \\
\text { premix }\end{array}$ & Salt \\
\hline $\begin{array}{c}\text { Digestible } \\
\text { energy }\end{array}$ & 3.85 & 3.23 & 4.05 & 4.25 & 3.4 & 3.44 & 7.7 & 0 & 0 \\
$\begin{array}{c}\text { Mcal/kg) } \\
\text { Crude protein }\end{array}$ & 9.4 & 17.2 & 49.9 & 68.5 & 44.9 & 37.8 & 0 & 0 & 0 \\
Arginine & 0.43 & 1.18 & 3.68 & 3.99 & 4.96 & 2.65 & 0 & 0 & 0 \\
Histidine & 0.29 & 0.49 & 1.38 & 1.94 & 1.27 & 1.06 & 0 & 0 & 0 \\
Leucine & 1.05 & 1.07 & 3.9 & 4.95 & 2.64 & 2.56 & 0 & 0 & 0 \\
Lsoleucine & 0.31 & 0.54 & 2.28 & 2.8 & 1.39 & 1.45 & 0 & 0 & 0 \\
Methionine & 0.2 & 0.27 & 0.72 & 1.92 & 0.71 & 0.71 & 0 & 0 & 0 \\
lysine & 0.27 & 0.7 & 3.13 & 5.24 & 1.85 & 2.12 & 0 & 0 & 0 \\
Threonine & 0.33 & 0.56 & 1.99 & 2.88 & 1.45 & 1.67 & 0 & 0 & 0 \\
Tryptophan & 0.07 & 0.24 & 0.63 & 0.72 & 0.54 & 0.55 & 0 & 0 & 0 \\
Phenylalanine & 0.43 & 0.69 & 2.62 & 2.73 & 2.38 & 1.53 & 0 & 0 & 0 \\
Valine & 0.38 & 0.78 & 2.34 & 3.3 & 1.9 & 1.79 & 0 & 0 & 0 \\
Calcium & 0.04 & 0.13 & 0.4 & 5.34 & 0.2 & 0.75 & 0 & 0 & 0 \\
Phosphorus & 0.3 & 0.18 & 0.71 & 3.05 & 1.15 & 1.1 & 0 & 0 & 0 \\
Maximum usage & 90 & 20 & 60 & 5 & 9 & 7 & 3 & 0 & 0 \\
Minimum usage & 20 & 0 & 10 & 0 & 0 & 0 & 0 & 0 & 0 \\
Equivalent usage & 0 & 0 & 0 & 0 & 0 & 0 & 0 & 1 & 0.3 \\
Price (Yuan/kg) & 1.5 & 1.5 & 2.8 & 6 & 1.6 & 1.4 & 6 & 10 & 0.8 \\
\hline
\end{tabular}


In order to facilitate the research, the data of feed materials and their nutritional composition and the feeding standard data of pigs in this paper are all selected from the NRC standard of the United States [8-9], and the price of raw materials adopts the average price of a certain place. The main raw materials and their nutritional composition [10], maximum and minimum limits of usage and prices of pig feed formulations were shown in Table 1.

In this paper, the feed formulation of pigs at the growth stage of 50-80kg was taken as the research objective, and the nutritional standards of pigs at this stage were shown in Table 2.

Table 2. Nutritional standard for pig feed of $50 \sim 80 \mathrm{~kg}$.

\begin{tabular}{cc}
\hline Nutrient & Nutritional standards \\
\hline Digestible energy (Mcal/kg) & 3.4 \\
Crude protein (\%) & 15.5 \\
Arginine (\%) & 0.27 \\
Histidine (\%) & 0.24 \\
Isoleucine (\%) & 0.42 \\
Leucine (\%) & 0.71 \\
Lysine (\%) & 0.75 \\
Methionine (\%) & 0.2 \\
Phenylalanine (\%) & 0.44 \\
Threonine (\%) & 0.51 \\
Tryptophan (\%) & 0.14 \\
Valine (\%) & 0.52 \\
Calcium (\%) & 0.5 \\
Phosphorus (\%) & 0.45 \\
\hline
\end{tabular}

\subsection{Feed Formula Model of Pig}

Pig feed formula design is a typical combination optimization problem, is different kinds of feed raw materials in proportion to a reasonable match to meet the specific nutritional needs of pigs at different weight stages and the lowest economic cost Eq. [7].

If $\mathrm{M}$ kinds of feed raw materials and $\mathrm{N}$ kinds of nutritional requirements are provided, the matrix $\mathrm{Pij}(1 \leq \mathrm{i} \leq \mathrm{n}, \quad 1 \leq \mathrm{j} \leq \mathrm{m})$ of $\mathrm{N}^{*} \mathrm{M}$ can be formed, as follows:

$$
P=\left[\begin{array}{ccc}
\mathrm{a}_{11} & \cdots & a_{1 m} \\
\vdots & \ddots & \vdots \\
a_{n 1} & \cdots & a_{n m}
\end{array}\right]
$$

Set variable $\mathrm{Z}$ as the economic cost of feed, then the mathematical model of pig feed formula is as follows:

Objective function:

$$
\min Z=c_{1} x_{1}+c_{2} x_{2}+\ldots+c_{n} x_{n}
$$


In Eq. (2), $\mathrm{c}_{i}$ is the price of various raw materials, and variable $x_{i}$ is the amount of various raw materials in the formula (in percentage).

The constraint conditions of feed on nutrient composition are expressed by inequality group:

$$
\left\{\begin{array}{l}
\mathrm{a}_{11} x_{1}+a_{12} x_{2}+\cdots+a_{1 n} x_{m} \geq b_{1} \\
\mathrm{a}_{21} x_{1}+a_{22} x_{2}+\cdots+a_{2 n} x_{m} \geq b_{2} \\
\vdots \\
a_{m 1} x_{1}+a_{m 2} x_{2}+\cdots+a_{m n} x_{m} \geq b_{n} \\
x_{i} \geq 0(i=1,2, \cdots, n) \\
\sum_{i=1}^{n} x_{i}=100
\end{array}\right.
$$

In Eq. (3), $a_{i j}$ is the hundredth component of the $\mathrm{j}$-th nutrient contained in the $\mathrm{i}$-th raw material; $\mathrm{b}_{i}$ is the content of each nutrient in the feeding standard.

\section{Solution to Feed Formula Model}

\subsection{Linear Programming}

When the linear programming method is used to solve the pig feed formula, it converts the relevant elements and constraints in the feed formula into linear mathematical functions, and solves the minimum value of the function under certain constraints [11]. In order to facilitate the operation, this paper uses the Linprog function that comes with Matlab to solve the linear programming to optimize the related problems.

The format of the Linprog function is as follows:

$$
[\mathrm{x}, \mathrm{favl}]=\operatorname{lingprog}(\mathrm{c}, \mathrm{A}, \mathrm{b}, \mathrm{Aeq}, \mathrm{beq}, \mathrm{vlb}, \mathrm{vub})
$$

In Eq. (4), $\mathrm{x}$ is the optimal solution; favl is the optimal value of the objective function; $\mathrm{c}$ is the coefficient of the objective function; $\mathrm{A}$ is the coefficient matrix of the inequality constraint; $b$ is the constant term of the inequality constraint; Aeq is the coefficient matrix of the equality constraint; beq is the constant term of the equality constraint; vlb is the lower bound of the variable; vub is the upper bound of the variable.

\subsection{Particle Swarm Optimization}

The basic idea of the particle swarm optimization algorithm originates from the research on the foraging behavior of bird flocks. Bird flocks adjust their search paths through their own experience and information sharing between the flocks, so that the flocks can find the optimal destination.

A kind of particle is used to simulate a bird in the search space, and the position of each particle corresponds to the potential solution of the optimization problem. In the search process, particles are described by position and velocity vectors, where the 
position vector represents the possible solution of the problem, and the velocity vector represents the direction and magnitude of the particle position change. Evaluate the fitness value of each particle through the set objective function, and iterate continuously until the optimal solution is found. The mathematical model of particle swarm optimization algorithm is as follows:

Suppose N particles search for the optimal solution in the d-dimensional space, and the position vector of the $\mathrm{i}$-th particle in the space can be expressed as:

$$
x_{i}=\left(x_{i 1}, x_{i 2}, \ldots, x_{i D}\right), \mathrm{i}=1,2, \ldots, n
$$

Its velocity vector can be expressed as:

$$
\mathrm{v}_{\mathrm{i}}=\left(v_{i 1}, v_{i 2}, \ldots, v_{i D}\right), i=1,2, \ldots, n
$$

The optimal position currently searched by the ith particle is called the individual extreme value, which can be expressed as:

$$
\text { pbest }_{\mathrm{i}}=\left(p_{i 1}, p_{i 2}, \ldots, p_{i D}\right), i=1,2, \ldots, n
$$

The optimal position currently searched by the entire population is called the global extreme value, which can be expressed as:

$$
\text { gbest }=\left(p_{g 1}, p_{g 2}, \ldots, p_{g D}\right)
$$

Each particle updates its position and velocity according to these two extreme values. The update formula is as follows:

$$
\left\{\begin{array}{l}
v_{i d}=w^{*} v_{i d}+c_{1} r_{1}\left(p_{i d}-x_{i d}\right)+c_{2} r_{2}\left(p_{g d}-x_{i d}\right) \\
x_{i d}=x_{i d}+v_{i d}
\end{array}\right.
$$

In Eq. (5), $\mathrm{w}$ is the inertia weight coefficient; $c_{1}$ and $c_{2}$ are individual learning factors and social learning factors, respectively; ${ }^{r}, r_{2}$ are random numbers in the interval $[0,1]$; In general, for the efficiency and accuracy of the search, the position and velocity vector of the particles will be restricted by the boundary:

$$
x_{\min } \leq x_{i} \leq x_{\max }, \quad v_{\min } \leq v_{i} \leq v_{\max }
$$

\section{Simulation and Result Analysis}

According to the feed ingredients and their nutrient content in Table 1 and the pig feeding standards in Table 2, 13 feed materials such as corn, wheat bran, soybean meal, fish meal, cottonseed cypress, etc. were selected to prepare $100 \mathrm{~kg}$ pig feed.

\subsection{Particle Swarm Optimization Simulation Experiment}

In the experiment, set the population size $\mathrm{N}=50$, the maximum number of iterations $\mathrm{G}=500$, the inertia weight $\mathrm{w}=0.9$, and the learning factor $c_{1}=2, c_{2}=2$.In the feed formula model established in this paper, the lowest economic cost is the objective function, and the fitness value takes the objective function value. The algorithm termination condition is: the particle swarm system loops 500 times.

The flow chart of particle swarm optimization algorithm is shown in the figure 1. 
The optimization process of feed formulation by particle swarm optimization algorithm is shown in the figure 2 , where the ordinate represents the optimal individual fitness value, and the abscissa represents the number of iterations. It can be seen from the convergence curve that the convergence speed of the algorithm slows down around 30 iterations, and stabilizes after 50 generations. This solution is the optimal solution for the pig feed formula. The result shows that the objective function value is $166.8732,{ }_{1}$, $x_{2}, \ldots, x_{13}$ are 57.6771, 14.8748, 10.0000, 0, 1.5705, 7.0000, 0, 2.0000, 4.9216, 0, 0, $1.000,0.3000$ respectively.

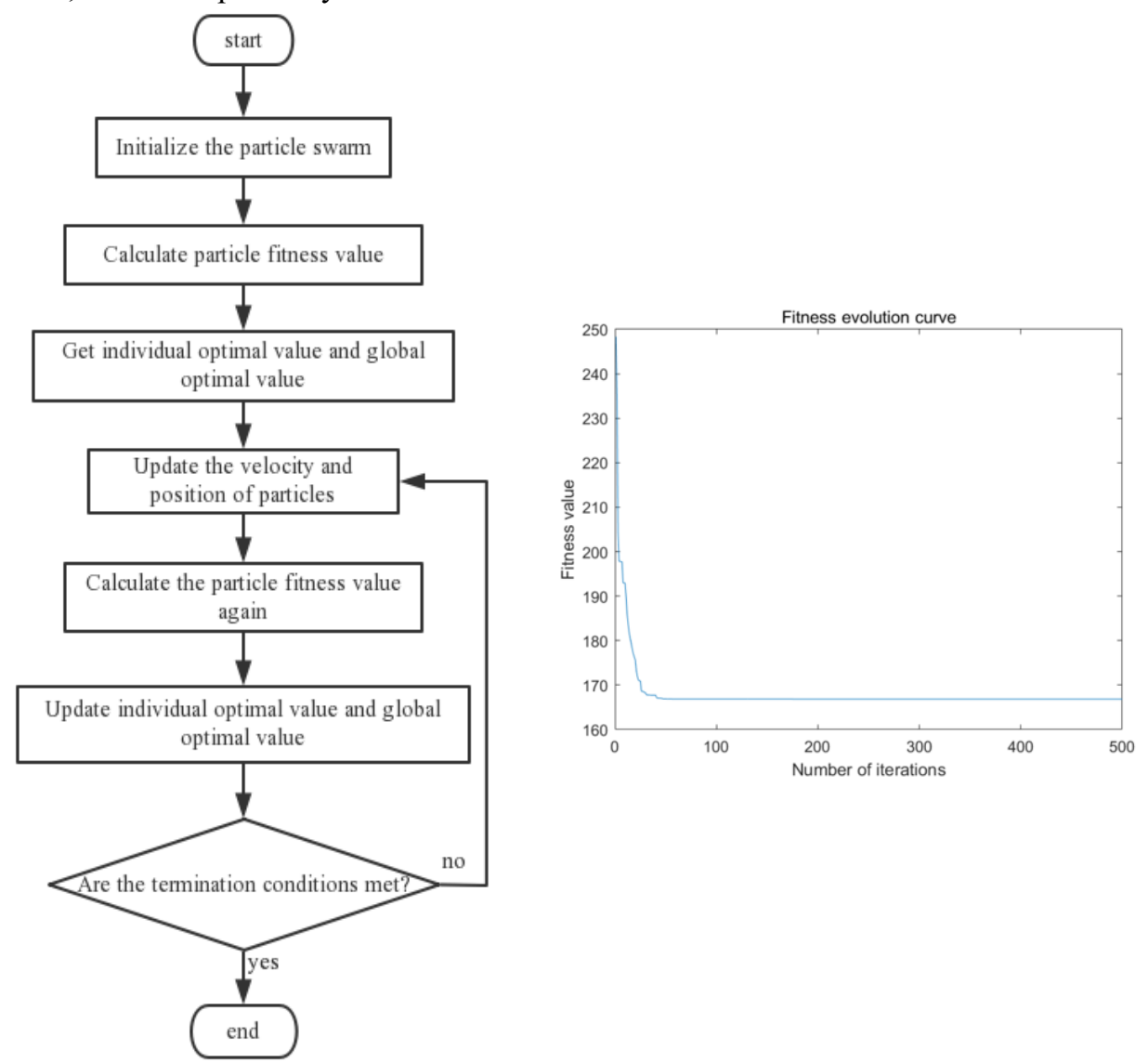

Figure 1. Control flow chart of particle swarm optimization algorithm.

Figure 2. Feed formulation optimization process.

\subsection{Comparison and Analysis}

Using particle swarm optimization algorithm and linear programming model respectively to solve the feed formulation, and the results are as follows, Table 3 and 4 are the output comparison of the two algorithms, the results show that, compared with linear programming, the cost of the feed formula obtained by particle swarm optimization algorithm is lower, and the nutrient elements in the formula are higher, also 
fully meet the constraints of 25 , that is, meet 14 constraints of nutritional requirements, and meet 11 constraints of upper and lower limits of usage.

Table 3. The cost comparison $(\mathrm{kg})$.

\begin{tabular}{lll}
\hline Formula composition & Particle swarm optimization & Linear programming \\
\hline Corn & 57.6771 & 54.7172 \\
Wheat bran & 14.8748 & 20.0000 \\
Soybean meal & 10.0000 & 10.0000 \\
Fish meal & 0 & 0 \\
Cottonseed meal & 1.5705 & 0.0467 \\
Rapeseed meal & 7.0000 & 7.0000 \\
Vegetable oil & 0 & 0 \\
Stone powder & 2.0000 & 2.0000 \\
Dicalcium Phosphate & 4.9219 & 4.9361 \\
Methionine & 0 & 0 \\
Lysine & 0 & 0 \\
Compound premix & 1.0000 & 1.0000 \\
Salt & 0.3000 & 0.3000 \\
total cost (yuan/100kg) & 166.8732 & 167.2723 \\
\hline
\end{tabular}

Table 4. The comparison of nutritional element standards (\%).

\begin{tabular}{cccc}
\hline Nutrient content & $\begin{array}{c}\text { Particle swarm } \\
\text { optimization }\end{array}$ & $\begin{array}{c}\text { Linear } \\
\text { programming }\end{array}$ & Standard requirement \\
\hline Digestible energy (Mcal/kg) & 3.4002 & 3.4000 & 3.40 \\
Crude protein & 16.3362 & 16.2604 & 15.50 \\
Arginine & 1.0549 & 1.0271 & 0.27 \\
Histidine & 0.4723 & 0.4695 & 0.24 \\
Leucine & 0.6105 & 0.6078 & 0.42 \\
Isoleucine & 1.3754 & 1.3590 & 0.71 \\
Methionine & 0.7503 & 0.7500 & 0.75 \\
Lysine & 0.2884 & 0.2855 & 0.20 \\
Threonine & 0.7571 & 0.7435 & 0.44 \\
Tryptophan & 0.6123 & 0.6091 & 0.51 \\
Phenylalanine & 0.1861 & 0.1881 & 0.14 \\
Valine & 0.7243 & 0.7241 & 0.52 \\
Calcium & 2.0193 & 1.9730 & 0.50 \\
Phosphorus & 1.1534 & 1.1385 & 0.45 \\
\hline
\end{tabular}

\section{Conclusions}

Particle Swarm Optimization (PSO) has been applied more and more in the field of combinatorial optimization, but there is little research on solving the problem of pig feed formulation. In this paper, a new method for solving the problem of feed formulation is presented. In this paper, a new method for solving the problem of feed formulation is presented. The model of feed formulation for pigs is established to optimize the feed formulation. The results are compared with those obtained by linear programming algorithm under the unified model. The results show that the particle swarm optimization algorithm has better results for the optimization of pig feed formula, lower economic cost and higher nutritional level, thus proving the advanced and effective of the algorithm. In addition, according to the different nutritional requirements of the pigs at different weight stages, the feed formula optimization plan can be quickly and conveniently generated, which is conducive to shortening the growth cycle of the pigs and saving economic costs, thereby further achieving precise feeding. However, this paper mainly 
takes economic cost as the goal. If we can combine the ammonia emission, lean meat ratio and other targets to carry out the feed ratio, a more optimized pig feed formula will be obtained.

\section{References}

[1] Zhang JX, Wu ZD. Application of particle swarm optimization technology in feed formulation design. Feed Industry 2010; 31(15): pp 47-48.

[2] Wang JR, Wang TP. Mathematical model of pig feed formula optimization based on intelligent algorithm China Feed 2021; 8: 145.

[3] Cao ZY. Application research of genetic algorithm in pig feed formula design (China: Yunnan University of Technology), 2016.

[4] Huang K. Research on the application of genetic algorithm in pig feed formula system, China: Soochow University, 2014.

[5] Feng J, Yue Q. An ant colony optimization algorithm for solving feed formula problem. J. Northeast Agricul. Uni. 2010; 41(03): 130-136.

[6] Liu ZH, Wei HL, Zhong QC et al. Parameter estimation for VSI-Fed PMSM based on a dynamic PSO with learning strategies. In: IEEE Trans. Power Electr. 2017; 32(4): 3154-3165.

[7] Yang SS. Research on optimized pig feed formula based on improved genetic algorithm (China: Anhui Agricultural University), 2019.

[8] Meng QX et al. Dairy Cow Nutritional Needs 7th Revised Edition (2001) China: China Agricultural University Publishing, 2002.

[9] Shiyan Q et al. Nutritional Needs of Pigs (Tenth Revised Edition, 1998), China: China Agricultural University Publishing, 1998.

[10] Huang K, Chang JY, Xie CH. Feed formula design based on NSGA-II Comp. Knowledge Technol. 2013; 9(20): 4692-4695.

[11] Zhang YC. Application of mathematical methods in feed formulation research and neural network evaluation of results. China: Inner Mongolia University, 2019. 